\title{
Can any anti-particle make a probe?
}

Positron probes (of the solid state) are already a booming business and may soon be applied to the study of liquids. Can anti-proton probes be far away?

EACH new particle of matter discovered may most immediately be of interest to those with theories to check (or invent, as the case may be), but one thing is certain: somebody will eventually use the particle as a probe for some purpose or another. And that is likely to be true even if the newly discovered particle is inherently unstable, or if it interacts with and is destroyed by some component of the environment in which it finds itself. What matters is merely that the particle should last for longer than the timescale of the phenomena studied. And the instability may then be an advantage, making plain either where the particle was before it ceased to exist or some other attribute of its state of being.

This generalization is best justified by the growth, in the past three decades, of the use of the positron as a probe, mostly of the solid state. It is an improbable development. Although positrons are inherently stable, with almost as much right as ordinary negatively charged electrons to continue indefinitely to exist, the whole world knows that positrons readily annihilate with electrons, so that their lifetime in the real world is best measured in, say, picoseconds $\left(10^{-12}\right.$ seconds). By way of compensation, the mutual annihilation of a positron and an electron yields two photons with a characteristic energy that peaks at $51.1 \mathrm{keV}$ - next to the $21-\mathrm{cm}$ line of hydrogen, the radiation frequency at which cosmologically inclined astrophysicists are always on the look-out, in observations of the galactic centre, for example.

Hitherto, the solid state has been the chief beneficiary. The principles are quite simple, at least on Page One. The yardstick of stability for a positron in the real world is the lifetime of positronium - the hydrogen 'atom' in which a positron has replaced the proton. Naturally, the singlet state (in which the spins are anti-parallel) is the least stable, decaying in 125 picoseconds. Any intrinsically more rapid phenomenon is in principle observable.

Naturally, there are complications, practical and of interpretation. Making nearly mono-energetic beams of positrons whose energy compares with those characteristic of the processes in solids is not child's play; the favoured techniques do not rely on high-energy physics, but on artificially produced isotopes such as ${ }^{22} \mathrm{Na}$ or ${ }^{58} \mathrm{Ni}$, which are positron emitters. But, as with $\beta$-emitters, these materials yield particles with a Fermi spectrum ranging from zero to some maximum.

The trick of making a mono-energetic beam of reasonable but still low energy usually entails some interaction with a solid within which the energy of incident positrons is first reduced to the average thermal energy, whereupon some proportion of the incident particles is afterwards re-emitted by scattering, but with an energy that is a measure of the work function - the potential difference between the interior and the exterior of the solid. Then, usually, there must follow devices for accelerating and shaping the beam. All that is intricate stuff.

And there is worse to come. Positrons fired into a solid are usually thermalized within a few picoseconds, whereafter several things can happen. The endpoint of a positron's existence is the formation of positronium, but until that happens it can diffuse. And what, then, is measured? The standard diagnostic appears to be the Doppler shift from the frequency of $511-\mathrm{keV}$ caused by the momentum of the positron, itself dominated (in metals, at least) by the energy of electrons in the conduction band. But since there are many incident positrons, the trick is to measure the shape of a photon-emission band centred on $511 \mathrm{keV}$ and then to make sense of it.

Even so, positrons not immediately captured are likely to be trapped at simple ionic vacancies in the crystal lattice of a metal. This process is efficient because the quantum wavelength of a positron thermalized to, say, room temperature is still many times the typical lattice spacing in solids. And, once trapped in a vacancy, the wave function of the positron will collapse geometrically as it substitutes for the missing ion, the chances of its forming positronium will decrease and, when it does link up with an electron from the solid, the momenta of both particles will be so small that two gamma rays eventually emitted in the annihilation will almost exactly compensate in momentum, so that their Doppler shift will be only small.

That explains the interest of positrons as probes in the exploration of defects in solids and of the detailed structure of surfaces. Different levels in a solid can be probed by different choices of incident energy. But it is only right to acknowledge that the hardy band of men and women working in this field are among those who most valiantly extract meaning from fuzzy data. Each of them deserves some kind of medal.
So what is new? Two things, both in the same issue of Physical Review Letters (2 September). First, E. Gramsch, K.G.Lynn, J. Throwe and I Kanazawa, from the Brookhaven National Laboratory, have extended the use of positrons as probes from solids to liquids, and with surprising results $(67,1,282 ; 1991)$. The incentive has been to see whether positron probes can throw light on the structure of liquids, for which purpose they have studied positron annihilation in gallium and bismuth at the melting transition.

One expectation is readily confirmed. At the transition from solid to liquid, the diffusion length of positrons in either $\mathrm{Ga}$ or $\mathrm{Bi}$ is abruptly decreased (by a factor 10 in the former and 3 in the latter). Simplemindedly, this means that, in a liquid, there are fewer special sites at which positrons may hide from contact with electrons. The surprise is that, in molten gallium, the diffusion length appears to increase exponentially with increasing temperature, and not linearly as in bismuth.

The explanation offered is that positrons in liquid gallium are trapped in temporary potential wells formed by fluctuations of ionic structure and then, when that disappears, hop to the next nearest such fluctuation. What matters is that the wavelength of the positron is still quite long, but the picture cries out for a little calculation.

And what else? If the charge-counterpart of the electron can be a probe, why not also the charge-counterpart of the proton, the anti-proton? It is too soon to say that that has been done, but a Japanese group seems to be on the way. M. Iwasaki et al. from the University of Tokyo used a beam of anti-protons from the TRISTRAN accelerator at the National Laboratory for High-Energy Physics at Tsukuba to look for long lived anti-protons trapped in liquid helium $(67,1,246 ; 1991)$.

The outcome has been well-flagged, both theoretically and in experiments with negative pions. In principle, it should be possible for an anti-proton to replace an electron in a helium atoms, when its lifetime should be much longer than the usual few picoseconds. What the Japanese find is that the annihilation of 3.6 per cent of the particles stopped in liquid helium is delayed, in some cases for as long as 30 microseconds - a factor of a million or so. For the time being, the trick seems to work only with helium, but it can only be a matter of time before somebody makes a probe of it.

John Maddox 